\title{
Implementation of an Inductively Coupled EM Probe System for PD Diagnosis
}

\author{
Hee-Dong Kim*, Noh-Joon Park** and Dae-Hee Park ${ }^{\dagger}$
}

\begin{abstract}
In recent years, various types of partial discharge (PD) methods such as capacitive, inductive, electromagnetic, and acoustic coupling techniques have been developed for diagnosing rotating machines. An electromagnetic (EM) probe, which is an inductively coupled type of sensor, is required for detecting corona and internal discharges during off-line tests. In this study, a new technique for enhancing the measurement sensitivities for corona and internal discharge based on an EM inductive position sensor is proposed. An EM probe that winds wires around horseshoe-shaped and cylindershaped ferrites as helices is designed and optimized for the implementation of off-line PD monitoring of the stator winding of a rotating machine. The measurement system based on this design is implemented, and it is verified from the results of the experiment performed in this study that the probe provides similar performance as existing commercial products.
\end{abstract}

Keywords: Partial discharge (PD), Electromagnetic (EM) probe, Mn-ferrite, Stator winding, rotating machine, Cable joint, Inductively coupled sensor, Corona discharge, Internal discharge.

\section{Introduction}

Stator winding is the most vulnerable component that causes failures in rotating electric machines. In highvoltage rotating machines, partial discharges (PDs) occur due to thermal, mechanical, and electrical stresses and single or complex deterioration factors, which are caused by exterior environments, in which PD signals represent instantaneous pulse rising time and width.

In terms of the deterioration of the stator winding of a rotating machine, three discharges have been known as representative defects. Internal discharges are caused by the void existing inside the stator winding; corona discharges are caused by the concentration of electric fields at the edge of the conductive paint; and slot discharges are caused by a removed part of the conductive paint due to the vibration of winding wires inside the iron core.

These discharges are generated at various frequency bands and can be measured using a low-frequency PD measurement method (IEC 60270), a high-frequency PD measurement method (IEEE Std. 1434-2000), or an electromagnetic signal detection method at a microwave band.

A PD test is a method that measures PD signals generated from the supply of electric fields in the defect of an insulator, which is caused by the deterioration of the stator windings. It is possible to apply various analyses at time

$\uparrow \quad$ Corresponding Author: Fellow member of the KIEE, Depatment of Electrical and Information Communication Engineering,

Wonkwang University, Korea. (parkdh@wku.ac.kr)

* $\quad$ Member of the KIEE, Korea Electric Power Corporation (KEPCO) Research Institute, Korea. (hdkim@kepri.re.kr)

** Member of the KIEE, Department of Electrical and Information Communication Engineering, Wonkwang University, Korea. (njpark@wku.ac.kr)

Received: January 15, 2010; Accepted: October 13, 2010 and frequency domains for the detected signals. Thus, this test has shown high reliability in the examination and identification of locally generated insulation problems in a winding wire insulator compared to other methods.

Currently, various PD detection methods have been developed for diagnosing rotating machines. They are categorized by coupling techniques, such as capacitive coupling, inductive coupling, electromagnetic coupling, and acoustic coupling.

However, it is necessary to develop an EM probe that can determine the exact location of the defect within the stator winding in order to verify the degree of deterioration in some defects occurring at the stator winding of a rotating machine. As such, this study develops an inductively coupled EM probe system for identifying the exact defect position in the stator winding of a rotating machine under the off-line environment.

First, an EM probe is designed, and a prototype is fabricated using a ferrite core. The PD signals of the stator winding of a $6.9 \mathrm{kV}$ class rotating machine that has internal and corona discharge defects is then measured after verifying the feasibility of the PD signals detection at a cable joint that has artificial void defects.

In addition, the results of the test performed in this study are compared with the frequency, and phase characteristics, as well as sensitivity of a commercial high-frequency current transformer (HFCT) sensor using a spectrum analyzer and oscilloscope.

\section{System Configuration}

\subsection{EM Probe Design}

An inductive sensor allows the system to be designed for 
detecting PD signals, which are processed according to conductors. In particular, it is sensitive to magnetic fields and can adjust the amplitude of a signal by changing the number of winding turns of the helix since the magnitude of the output for given the PDs depends on inductance values.

Rogowski coils and current transformers are generally used as inductive sensors; the Rogowski coil has no iron cores and applies sensing for PD signals through the air, whereas the current transformer probe has iron cores and usually plays a role in enhancing its sensitivity. Current transformer probe, such as HFCT or radio frequency current transformer (RFCT) has also been commonly used in the diagnosis of high-voltage PD signals.

This study considers an EM probe that winds wires around ferromagnetic materials, which have high permeability, instead of using an iron core with a helical shape. As illustrated in Fig. 1, two different types of ferrite cores, horseshoe shaped (U shaped) and cylinder shaped, were considered for the EM probe designed to scan the defect position of the stator winding.

To optimize the design parameter of the EM probe, this study analyzed the characteristics of the distribution of magnetic fields around the ferrite. The shape of the ferrite, its permeability, and the number of turns in the helix were then optimized for concentrating the magnetic fields to the defect position of the stator winding. To enhance the inductively coupled current, 11.51 weight $\% \mathrm{Mn}$ ferrite $\left(\mathrm{MnFe}_{2} \mathrm{O}_{4}\right)$ was considered, as shown in Fig. 2.

In the detection of discharge signals, the probe size was determined in order to consider the interval of the stator winding. The fabrication was then carried out using a 0.6 $\mathrm{mm}$ thick wire for 11 and 7 turns of winding as a helical shape. Table 1 shows the design parameter used in this fabrication.

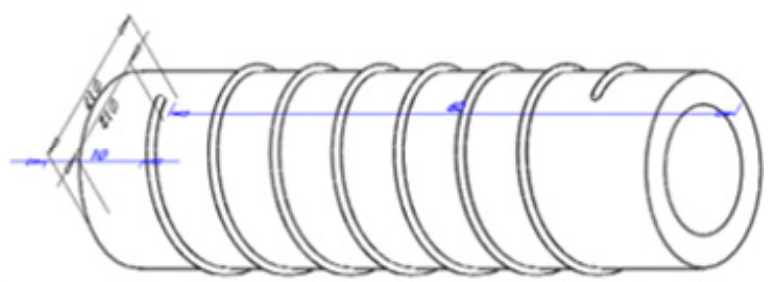

(a) Cylinder-shaped core

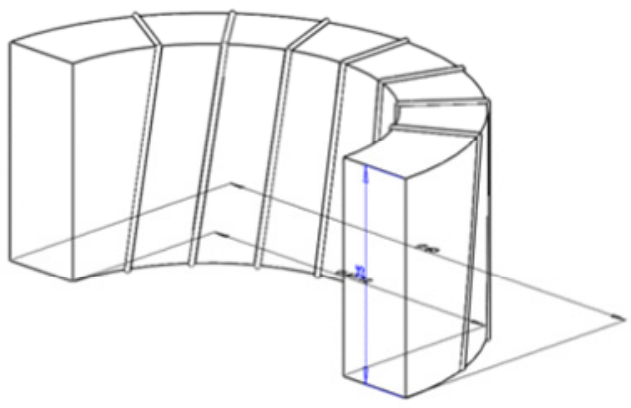

(b) Horseshoe-shaped core

Fig. 1. Configuration of the EM probe.
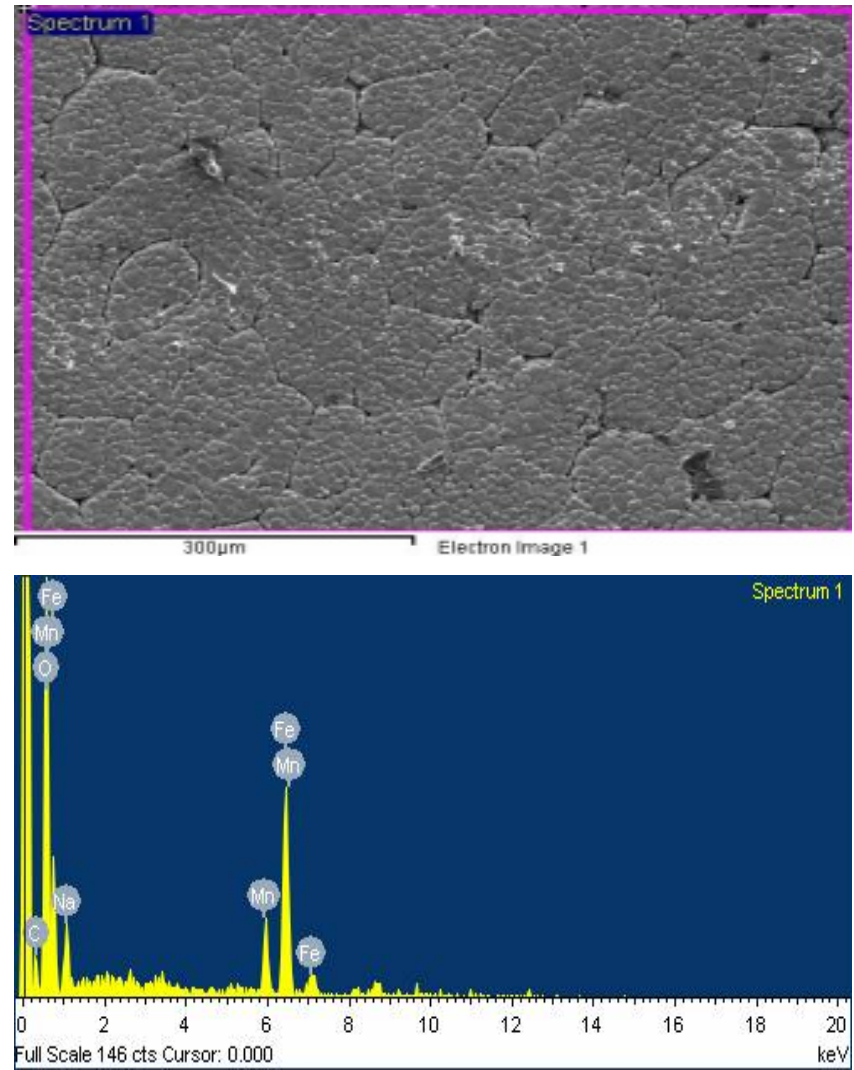

Fig. 2. SEM analysis of Mn ferrite used in the probe.

Table 1. Design parameters of the proposed EM probe

\begin{tabular}{c|c|c|c}
\hline \multicolumn{2}{c|}{ Parameters } & $\begin{array}{c}\text { Horseshoe } \\
\text {-shaped core }\end{array}$ & $\begin{array}{c}\text { Cylinder } \\
\text {-shaped core }\end{array}$ \\
\hline \multirow{4}{*}{ Ferrite } & Outside [mm] & 60 & 17 \\
\cline { 2 - 4 } & Inside [mm] & 40 & 12 \\
\cline { 2 - 4 } & Height [mm] & 25 & 60 \\
\cline { 2 - 4 } & Permeability & 2400 & 2400 \\
\hline \multirow{2}{*}{ Coil } & Thickness [mm] & 0.6 & 0.6 \\
\cline { 2 - 4 } & Turns & 11 & 7 \\
\hline
\end{tabular}

\subsection{Measurement System}

Because the fabricated EM probe was designed based on an inductive position sensor that detects PD signals through sensing the change in inductances caused by the current at the conductors, it can be used not only in detecting the stator winding of a rotating machine but also in the PD signals of power cables. Thus, it is a measurement system configured for applying the fabricated EM probe to each case and analyzing the results of the measurement.

First, this study configured the measurement system as shown in Fig. 3 in order for it to be applied in the diagnosis of PD signals in the joint box of XLPE power cables.

The measurement system consists of a power supply equipment (Hipotronix 750-2CTS) that can supply a maximum voltage of $50 \mathrm{kV}$ to the leading end of the power cable; a spectrum analyzer (Agilent N9320A); an oscilloscope (Tektronix TDS-3032); fabricated horseshoe-shaped and cylinder-shaped probes, HFCT, and other elements. 


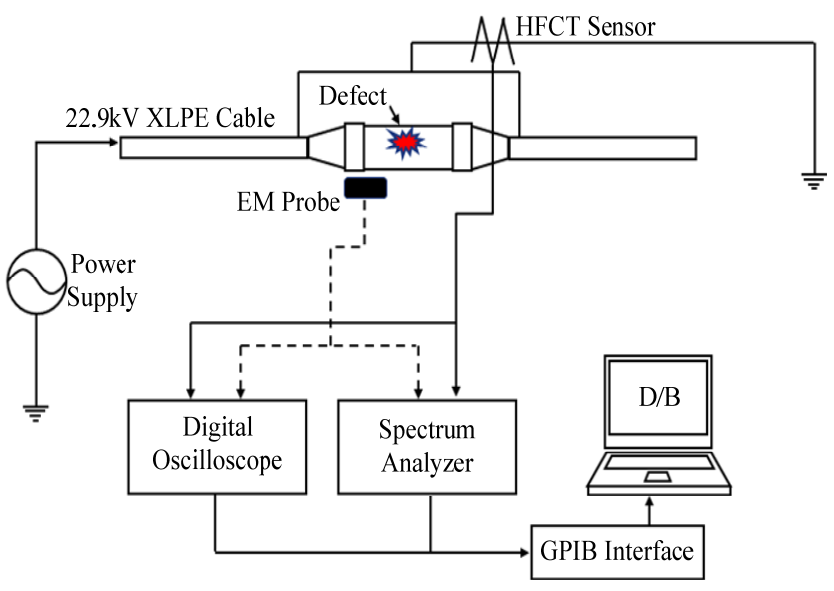

Fig. 3. Diagnosis system for XLPE cable joint.

The power cables were a distribution class of TR-CNCV-W $325 \mathrm{~mm}^{2}$, and two cables measuring $7 \mathrm{~m}$ and $6 \mathrm{~m}$ were connected using a joint box used in the joint of distribution cables in which artificial void defects were determined inside the joint box.

When the voltage was raised from $0 \mathrm{kV}$ to $20 \mathrm{kV}$ by AC power supply, $\mathrm{PD}$ signals were measured at the joint box of XLPE power cables with $15 \mathrm{kV}$. The fabricated horseshoeshaped and cylinder-shaped probes were used to detect PD signals by placing them to the joint box. In addition, the HFCT used as a reference sensor for comparing the results was set up in neutral line grounded to the joint box. The results of the measurement were analyzed based on frequency and phase domain analysis methods using a spectrum analyzer and oscilloscope.

In addition, the designed EM probe for scanning the defect position of the stator winding used ferrite, which has a permeability of 2,400 , as a core, with its shapes being the same as those of the diagnosis of power cables, such as horseshoe-shaped and cylinder-shaped cores. Moreover, the size of the probe in the detection of discharge signals was determined according to the interval applied to the stator winding. In the fabrication, $0.6 \mathrm{~mm}$ thick wires were coiled by 11 and 7 turns to the ferrite core as a helix shape.

In terms of the method used in the measurement system, the proposed EM probe was used around the stator winding while a high voltage is applied; scanning was then performed for the detected position. The phase and frequency characteristics presented in the results of the measurement were analyzed using a spectrum analyzer (sweep time : 3 seconds) and an oscilloscope. The HFCT sensor was also set up to the ground of the stator winding under the same condition in order to verify the sensitivity of the EM probe and compare the PD signals.

The stator winding used in the test has corona and internal defects, wherein PDs occurred at $6.5 \mathrm{kV}$ of the supplied high voltage. Fig. 4 illustrates the configuration of the measurement system for scanning the PD signal occurring at the stator winding. Fig. 5 shows the stator winding and the measurement system of the rotating machine disassembled for the dismantled diagnosis.

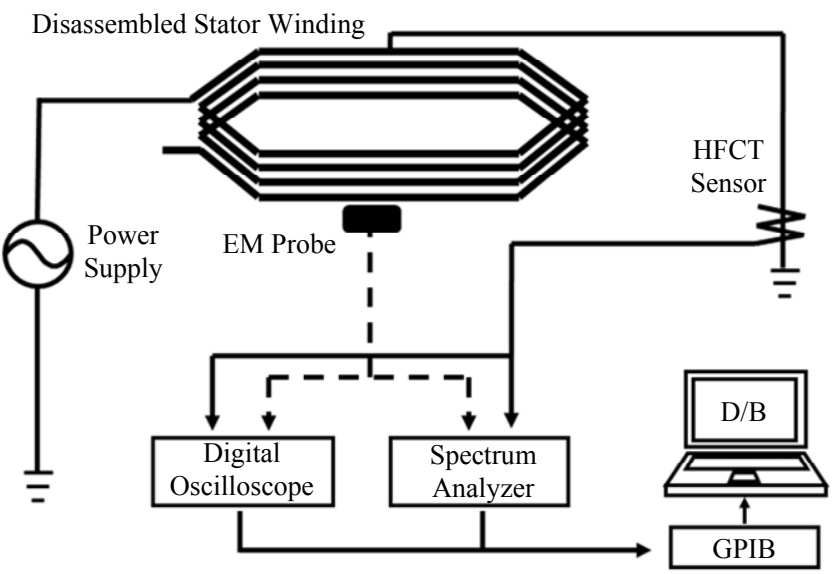

Fig. 4. Configuration of the stator winding diagnosis system with the proposed EM probe.

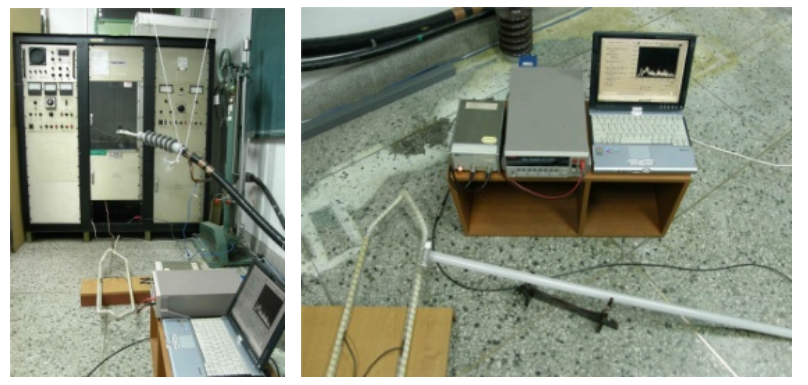

Fig. 5. Disassembled stator winding and the implemented measurement system for the dismantled diagnosis.

\section{Experimental Results}

Fig. 6 shows the HFCT sensor for the voltage supply of $15 \mathrm{kV}$ and the phase characteristics according to the number of turns in the EM probe fabricated using horseshoeshaped ferrite core.

The phase characteristics measured by the HFCT sensor represent a signal pattern of typical internal discharges with a $180^{\circ}$ phase difference. The fabricated EM probe also shows the same internal discharge patterns.

However, in consideration of the characteristics of the internal discharge measured in this study, the number of pulses measured by the EM probe was less than that of the HFCT sensor; however, the maximum amplitudes of the discharge signal in the EM probe recorded larger values of about 1.4 times, i.e., $0.35 \mathrm{~V}$, compared with that in the HFCT sensor. Meanwhile, there were no significant changes in the sensitivity based on the given number of turns in the helix of the EM probe with horseshoe-shaped ferrite core.

Fig. 7 shows the comparison of the measurement results of the phase characteristics between the EM probe with cylinder-shaped ferrite core and the HFCT sensor. As can be seen, there were internal discharge signals representing a $180^{\circ}$ phase difference, which is the same as the results of 


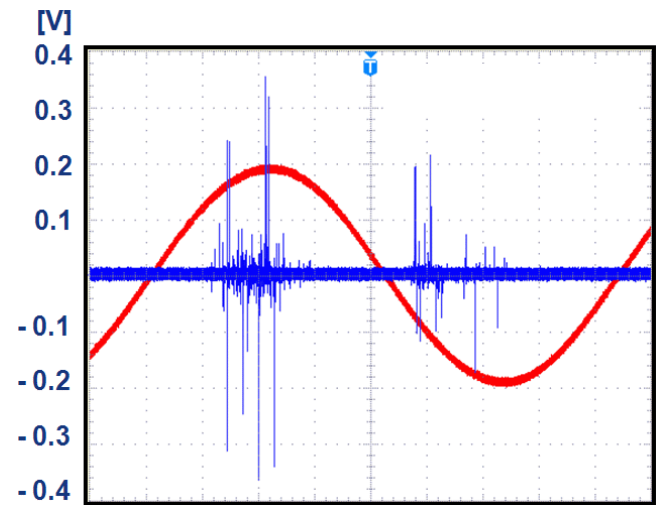

(a) 3-turn windings

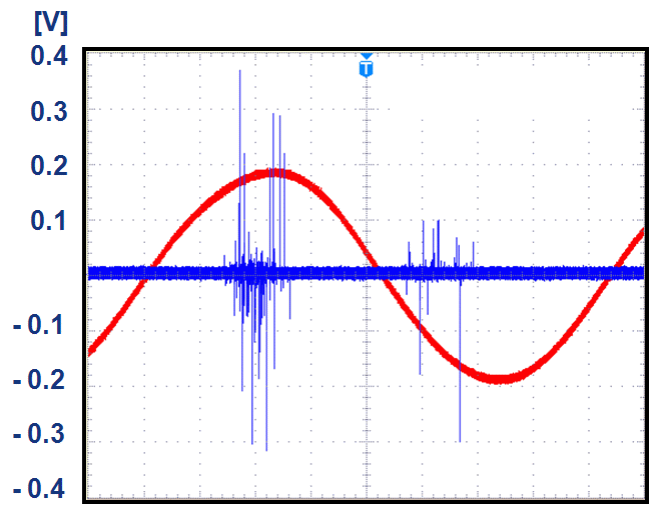

(b) 6-turns winding

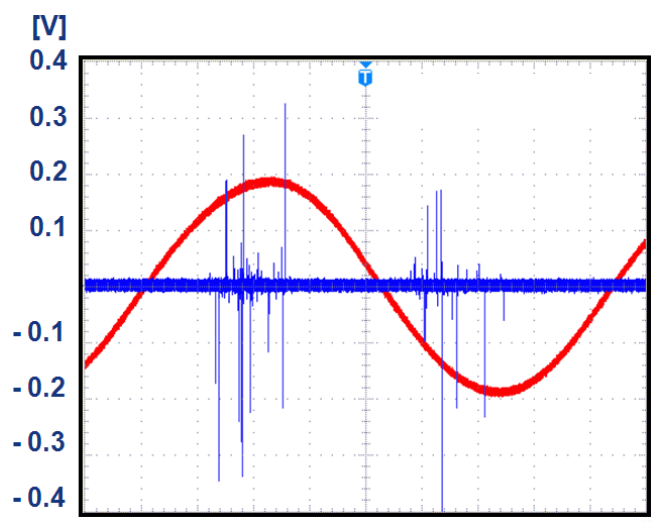

(c) 11-turn windings

Fig. 6. Phase characteristics of the EM probe with the horseshoe-shaped ferrite core.

comparison with the horseshoe-shaped ferrite core.

In addition, although the internal discharge detected by the EM probe with cylinder-shaped ferrite core showed a decrease in the number of pulses compared with that with horseshoe-shaped ferrite core, the maximum amplitudes of the signal in the EM probe represented larger values of about 2 times, i.e., $0.7 \mathrm{~V}$, compared with that of the horseshoe-shaped ferrite core, which was about 2.8 times compared with that of the HFCT sensor. The level of sensitivity according to the given number of turns in the helix showed no significant changes in value as that of the EM probe with horseshoe-shaped ferrite core.

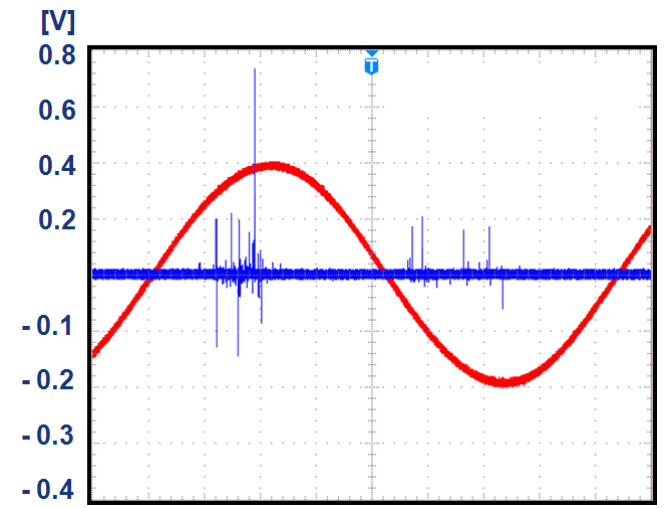

(a) 3-turn windings

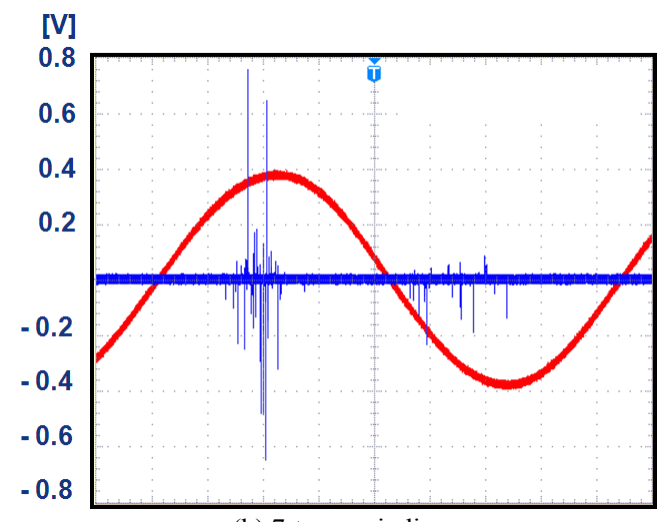

(b) 7-turns winding

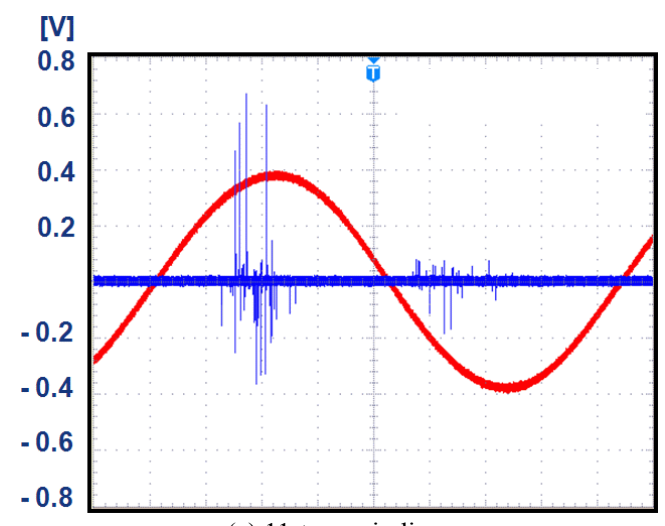

(c) 11-turn windings

Fig. 7. Phase characteristics of the EM probe with the cylinder-shaped ferrite core.

Fig. 8 shows the results of the frequency characteristics of the HFCT sensor and the EM probe with horseshoeshaped ferrite core according to the number of turns under the same condition, which were measured using a spectrum analyzer. It was verified that the commercial HFCT sensor is operated at a frequency band of below $50 \mathrm{MHz}$ based on $-50 \mathrm{dBm}$, whereas the fabricated horseshoe-shaped EM probe showed a wide band characteristic operated below $400 \mathrm{MHz}$ based on $-50 \mathrm{dBm}$.

The maximum signal amplitudes in the HFCT sensor were $-17 \mathrm{dBm}$ and $-30 \mathrm{dBm}$ for the bands within $10 \mathrm{MHz}$ and $15 \mathrm{MHz}$, respectively. Meanwhile, in the case of the 


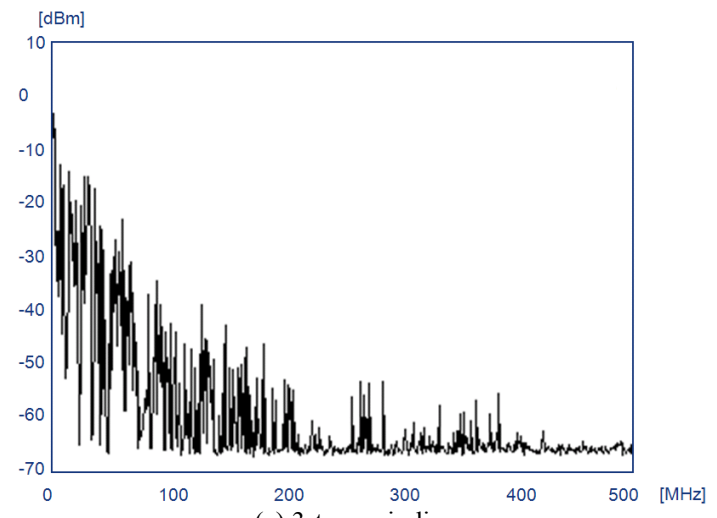

(a) 3-turn windings
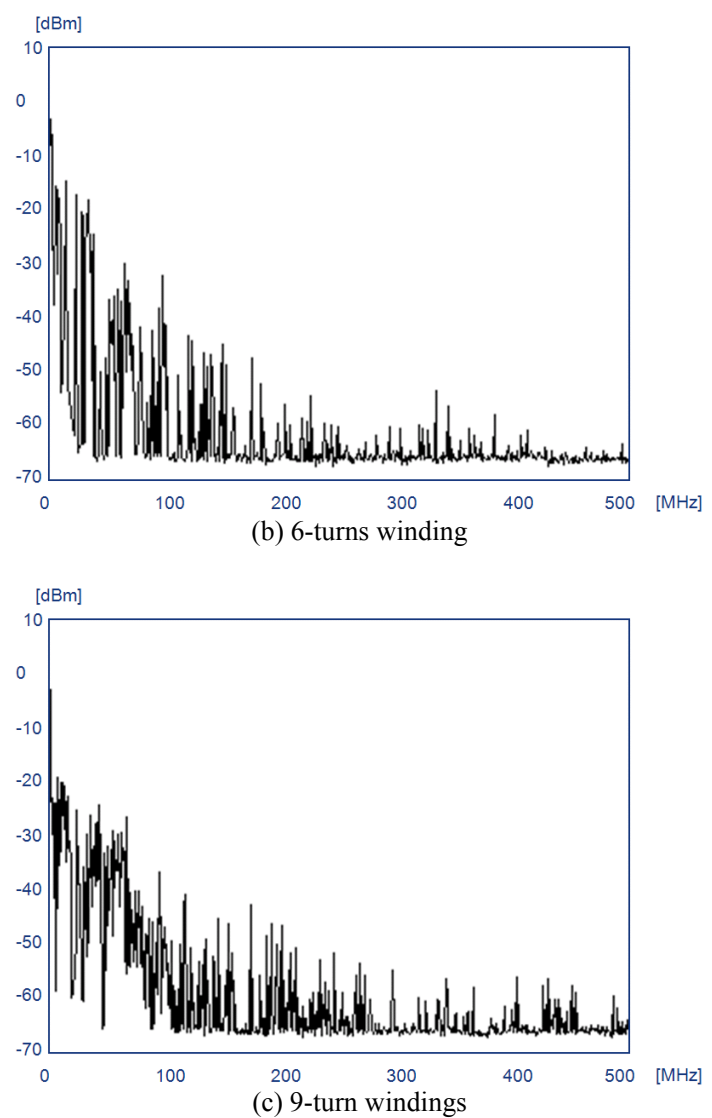

Fig. 8. Frequency characteristics of the EM probe with the horseshoe-shaped ferrite core at $500 \mathrm{MHz}$.

horseshoe-shaped EM probe with three and six turns, the maximum signal amplitude was $-15 \mathrm{dBm}$ for bands within $30 \mathrm{MHz}$, and $-20 \mathrm{dBm}$ for the probe with nine turns within such band. In the case of the horseshoe-shaped ferrite core, the maximum signal magnitude at $50 \mathrm{MHz}$ according to the number of turns was about $-25 \mathrm{dBm}$, which is a similar level. Thus, comparing the operation frequency band referenced on $-25 \mathrm{dBm}$, the EM probe with horseshoe-shaped ferrite core represented about $50 \mathrm{MHz}$, which was a wide band compared to that of the HFCT sensor.

Fig. 9 shows the results of the comparison of the frequency characteristics in the EM probe with cylinder-

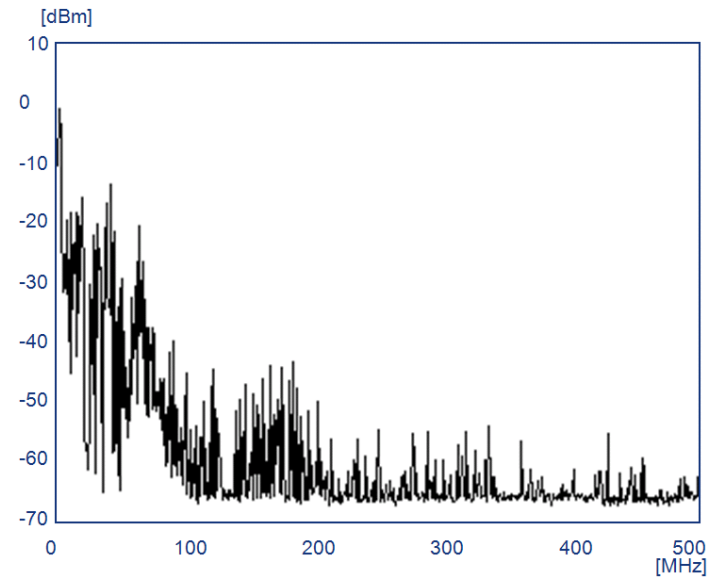

(a) 3-turn windings

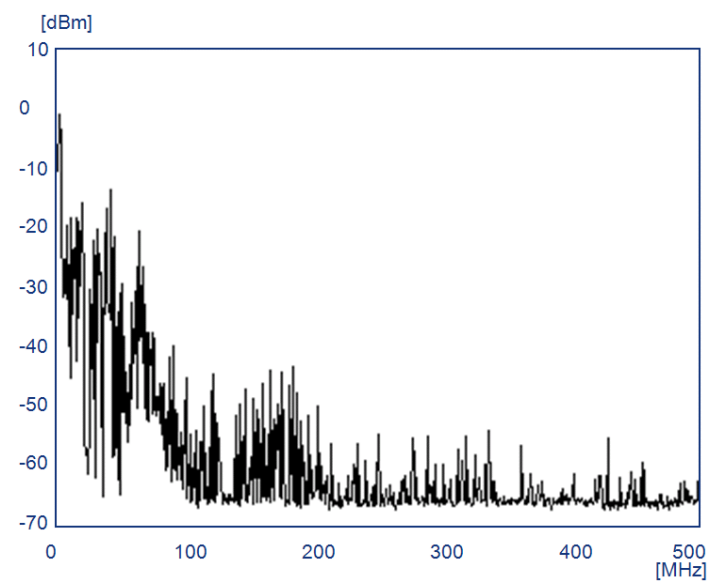

(b) 7-turns winding

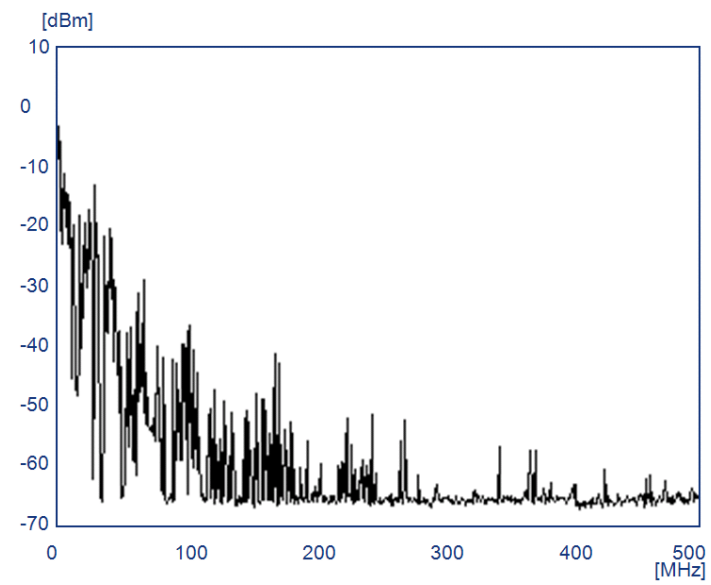

(c) 11-turn windings

Fig. 9. Frequency characteristics of the EM probe with the cylinder-shaped ferrite at $500 \mathrm{MHz}$.

shaped ferrite and the HFCT sensor. The maximum signal amplitude in the HFCT sensor was $-15 \mathrm{dBm}$ at a $10 \mathrm{MHz}$ band, while the cylinder-shaped EM probe showed -13 $\mathrm{dBm}$ at a frequency band of below $100 \mathrm{MHz}$, which was a similar pattern as that of the EM probe with horseshoeshaped ferrite core.

Therefore, the application of the EM probe with horse- 
shoe-shaped and cylinder-shaped ferrite cores to the diagnoses of the partial charge of power cables showed more excellent sensitivities than that of the HFCT sensor. In addition, the maximum detecting amplitude of the EM probe with cylinder-shaped ferrite shows about twice that of the EM probe with horseshoe-shaped ferrite core, indicating that sensitivity is not influenced by the number of turns in the helix but depends on the shape of the ferrite.

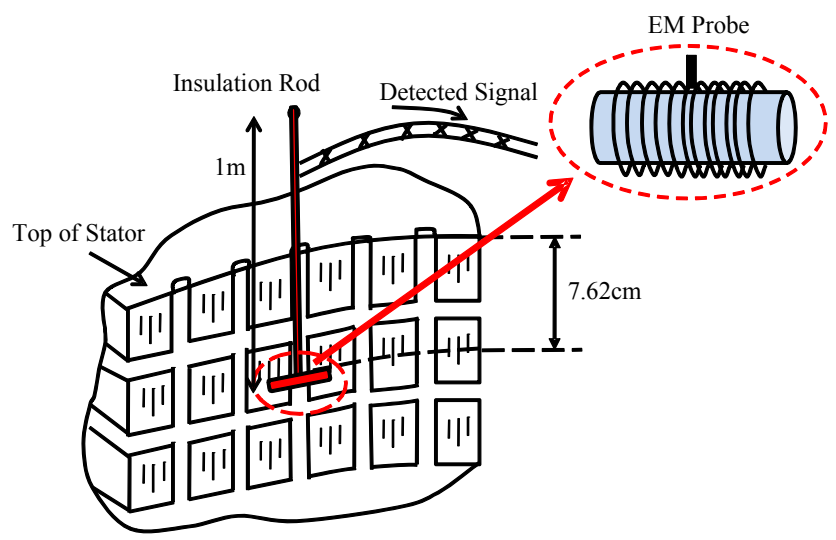

Fig. 10. Off-line diagnosis of the stator windings.

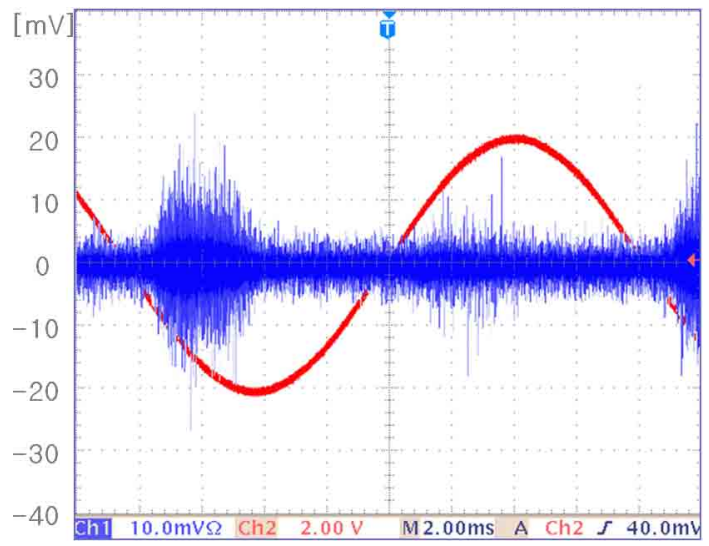

(a) Horseshoe-shaped core

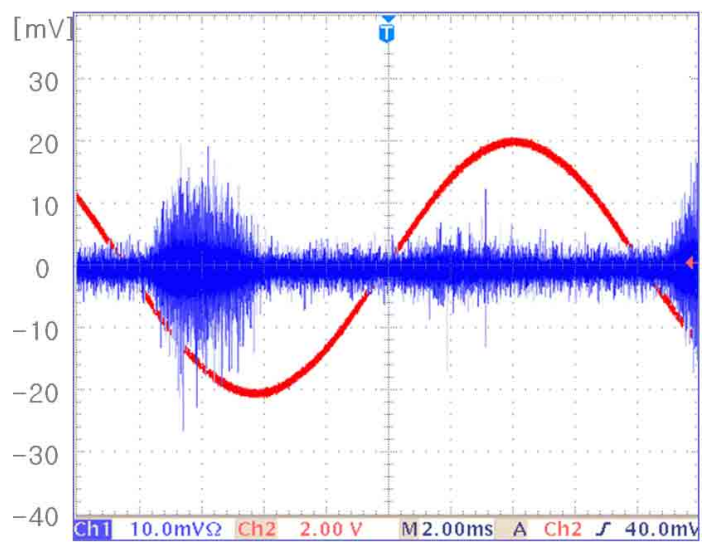

(b) Cylinder-shaped core

Fig. 11. Phase characteristics of the stator winding with corona discharge defect.

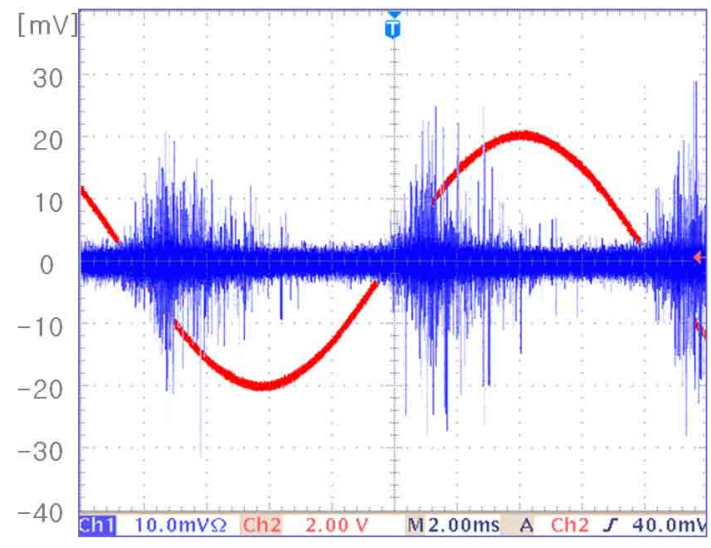

(a) Horseshoe-shaped core

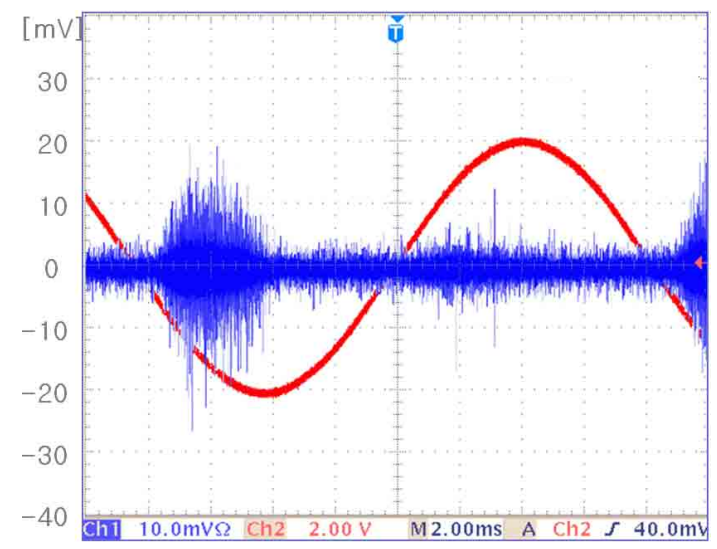

(b) Cylinder-shaped core

Fig. 12. Phase characteristics of the stator winding with internal discharge defect.

Fig. 10 shows the diagram of the off-line diagnosis of the stator winding. The detected inductive current can be measured using a current meter through a linear amplifier.

Figs. 11 and 12 illustrate the phase characteristics measured at the stator winding with corona and internal discharge defects when $6.5 \mathrm{kV}$ voltage was applied. In the phase characteristics of the measured PD signals, the corona discharge signals were detected in all cases, the HFCT sensor and the EM probe with the cylinder-shaped and horseshoe-shaped ferrite cores, at a $270^{\circ}$ phase under the same condition. In addition, internal discharge signals that occurred at a $180^{\circ}$ phase difference showed the same pattern.

The amplitude of the detected signals showed the same level at around $\pm 20 \mathrm{mV}$. In particular, the detected number of PD signals in the fabricated EM probe showed more excellent performance than that in the commercial HFCT sensor.

Figs. 13 and 14 represent the frequency characteristics of the PD signals measured using a spectrum analyzer in the stator winding with corona and internal defects, respectively. It was verified that the bands that showed increases in the signal amplitude of the PD that occurred at a high voltage supply of $6.5 \mathrm{kV}$ were $20-30 \mathrm{MHz}$ and $150-170$ $\mathrm{MHz}$. Table 2 shows the increasing range of the signals. 


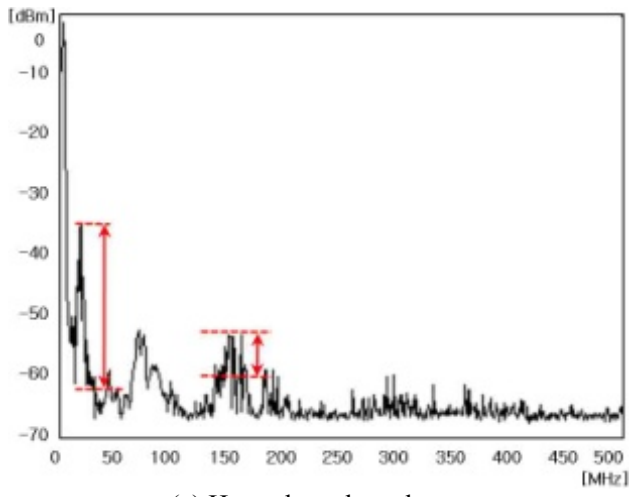

(a) Horseshoe-shaped core

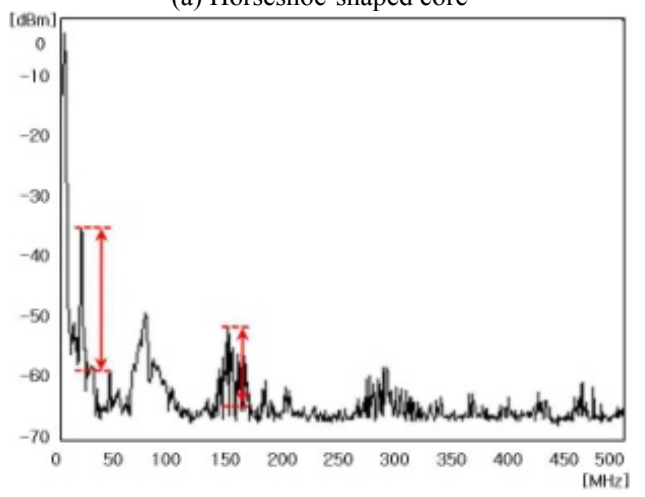

(b) Cylinder-shaped core

Fig. 13. Frequency characteristics of the stator winding with corona discharge defect.

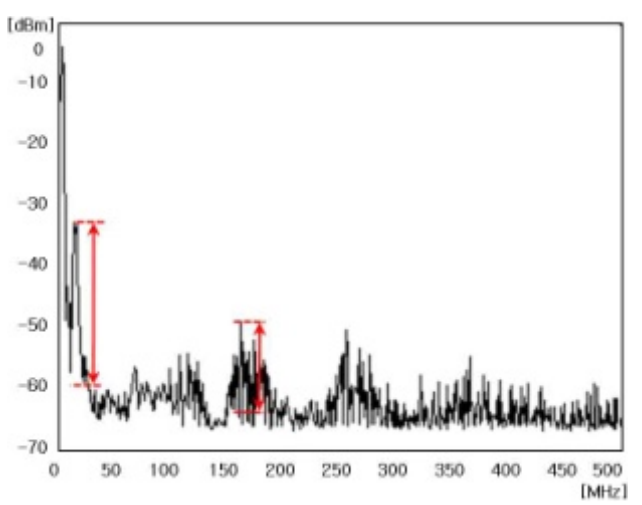

(a) Horseshoe-shaped core

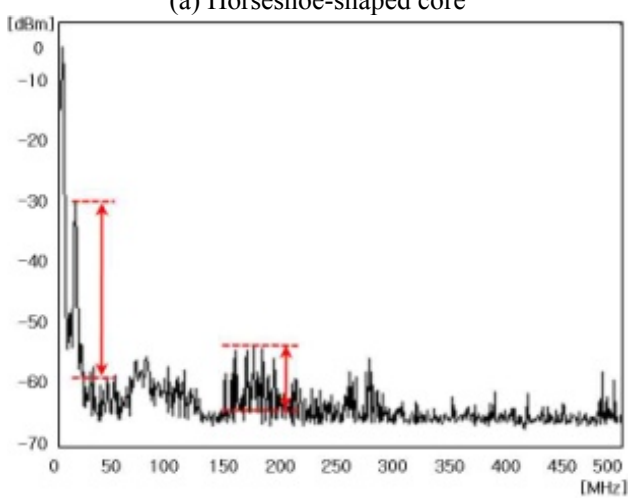

(b) Cylinder-shaped core

Fig. 14. Frequency characteristics of the stator winding with internal discharge defect.
Table 2. Comparison of the sensitivity of the EM probe

\begin{tabular}{c|c|c}
\hline $\begin{array}{c}\text { Increasing band of } \\
\text { the signal amplitude }\end{array}$ & $\begin{array}{c}\text { Corona discharge } \\
\text { defect }[\mathrm{dBm}]\end{array}$ & $\begin{array}{c}\text { Internal discharge } \\
\text { defect }[\mathrm{dBm}]\end{array}$ \\
\hline $\begin{array}{c}\text { Horseshoe-shaped } \\
\text { type } 20-30[\mathrm{MHz}]\end{array}$ & -26 & -27 \\
\hline $\begin{array}{c}\text { Horseshoe-shaped } \\
\text { type 50-170 [MHz] }\end{array}$ & -10 & -15 \\
\hline $\begin{array}{c}\text { Cylinder type } \\
20-30[\mathrm{MHz}]\end{array}$ & -26 & -28 \\
\hline $\begin{array}{c}\text { Cylinder type 50- } \\
170[\mathrm{MHz}]\end{array}$ & -15 & -12 \\
\hline
\end{tabular}

\section{Conclusions}

In this paper, an EM probe with horseshoe-shaped and cylinder-shaped ferrite cores was designed, fabricated, and investigated. The following characteristics were observed from the diagnosis tests performed on disassembled stator windings.

The performance (number of detected signals) of the fabricated EM probe was superior compared to that of a commercial HFCT sensor-based PD measurement system for an internal discharge with $180^{\circ}$ phase difference.

The detection performance of the fabricated EM probe was also more superior compared to that of a commercial HFCT sensor-based PD measurement system for a corona discharge that occurred at a $270^{\circ}$ phase. The horseshoeshaped EM probe represented the detected number of pulses with higher accuracy compared to that of the cylinder-shaped EM probe.

It was verified that both EM probes were operated at a wide frequency band of below $200 \mathrm{MHz}$. The amplitude of the detected signals increased at the ranges of $20-30 \mathrm{MHz}$ and $150-170 \mathrm{MHz}$.

Because the joint box of power cables was larger than the EM probe designed in this study, the cylinder-shaped EM probe provided superior characteristics. Meanwhile, for the diagnosis of stator windings, the horseshoe-shaped EM probe provided superior characteristics due to higher concentration in the magnetic flux.

\section{Acknowledgment}

This research was supported by the Power Generation and Electricity Delivery of the Korea Institute of Energy Technology Evaluation and Planning (KETEP) grant funded by the Korea government's Ministry of Knowledge Economy. (No. R-2006-1-124-0003)

\section{References}

[1] IEC 60270, "High-voltage test techniques-Partial discharge measurements", pp. 25-37, 2000.

[2] IEEE Std 1434-2000, "IEEE Trail-Use Guide to the Measurement of PD in Rotating Machinery", pp. 229, 2000. 
[3] N.H. Ahmed, N.N. Srinivas, "Online Partial discharge detection in cables," IEEE Trans. on Dielectrics and Electrical Insulation, Vol. 5, No. 2, pp. 181-188. 1998.

[4] N. De Kock, B. Coric, R. Pietsch, "UHF PD detection in GIS- suitability and sensitivity of the UHF method in comparison with the IEC 270 method," IEEE Electrical Insulation Magazine, Vol. 12, No. 6, pp. 20-26, 1996.

[5] M. Kawada, "Ultra Wide Band VHF/UHF Radio Interferometer System for Detecting Partial Discharge Source," IEEE Power Engineering Society Winter Meeting, Vol. 2, pp. 1482-1487, 2002.

[6] N. C. Sahoo, M. M. A. Salama, and R.Bartnikas, "Trends in partial discharge pattern classification : A Survey", IEEE Trans. on Dielectrics and Electrical Insulation, Vol. 12, No. 2, pp.248-264, 2005.

[7] Denissov, Ruben Grund, Thomas Klein, Wolfgang, Stefan Tenbohlen, "UHF Partial Discharge Diagnosis of Plug-In Cable Terminations," Jicable, 2007.

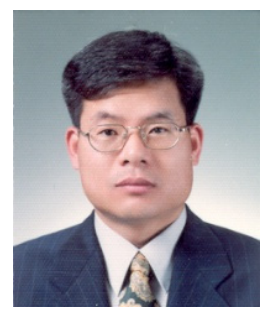

Hee-Dong Kim received his B.S., M.S., and Ph.D. degrees in Electrical Engineering from Hongik University, Seoul, Korea in 1985, 1987, and 1998, respectively.

Since 1990, he has been with Korea Electric Power Corporation (KEPCO) Research Institute, Daejeon, Korea, where he is currently a Principal Researcher. He was a Visiting Researcher with the Department of Electrical Engineering, Kyushu Institute of Technology, Kitakyushu, Japan. His research interests include aging mechanisms, diagnostic tests, partial discharge testing, and life assessment for rotating machines, and cable insulation systems.

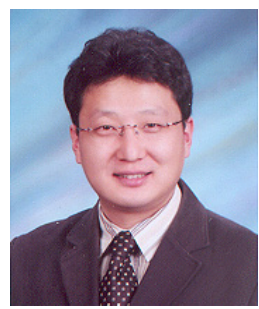

Noh-Joon Park received his B.S., M.S., and Ph.D. degrees in Electronics Engineering from Wonkwang University in 1993, 1995, and 2004, respectively.

Since 2006, he has been a Research Professor with Center for Advanced Electric Applications at Wonkwang University, working on design and analysis of EM sensors for partial discharge diagnosis in high-voltage engineering, electronic circuit design with impedance matching technique for light sources. His research interests include CMD and power IT, analog integrated circuit design with antenna system, channel measurements, and modeling of wireless communication system.

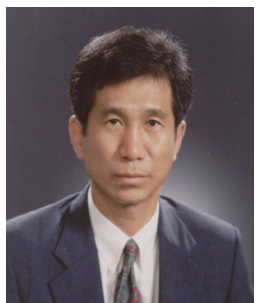

Dae-Hee Park was born in 1954 in Korea. He received his B.S. and M.S. degrees in Electrical Engineering from Hanyang University, Seoul, Korea in 1979 and 1983, respectively, and his Ph.D. degree from Osaka University, Osaka, Japan in 1989. He worked at the LG Cable Research Institute as a Senior Researcher from 1979 to 1991.

After that, he joined the School of Electrical, Electronics and Information Engineering at Wonkwang University where he is currently employed as a Professor. He has also worked as Director of the Center for Advanced Electric Application at Wonkwang University from 2004. He was at MSU in the USA as a Visiting Professor from 1999 to 2000. Since 2009, he is a Chairman at the College of Engineering, Wonkwang University. His main research interests are in the areas of insulting and dielectric materials, new lighting sources, and discharge. He is a member of the Korean IEE, Korean IEEME, and IEE Japan. 